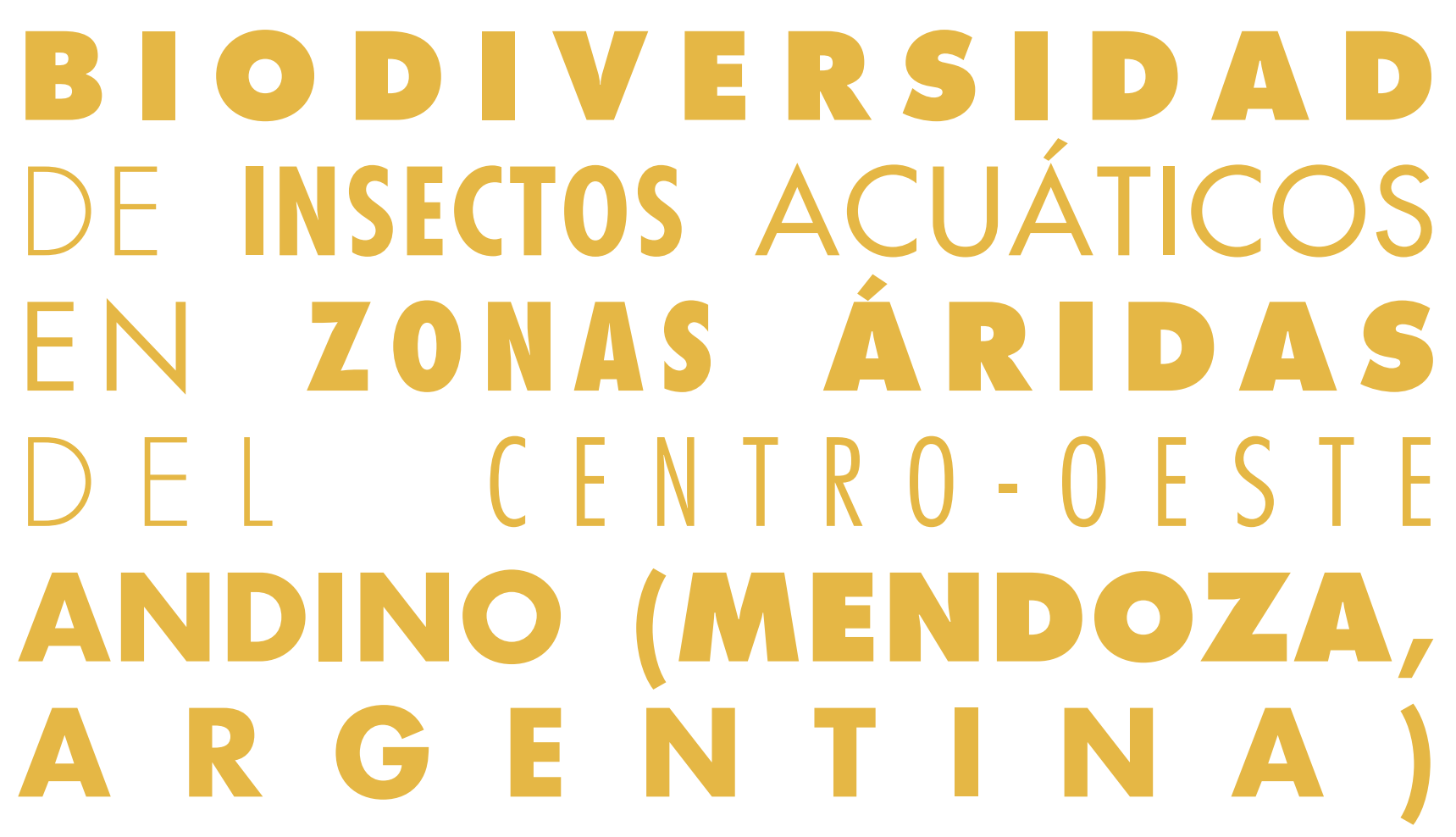

\title{
E. E. Scheibler
}

I.A.D.I.Z.A. Instituto Argentino de Investigaciones de Zonas Áridas escheib@lab.cricyt.edu.ar

\section{INTRODUCCIÓN}

La conservación de los ecosistemas y su biodiversidad han recibido mucho interés en los últimos años y actualmente es considerado un tema prioritario a nivel mundial. Los ecosistemas acuáticos revisten un especial interés para el ser humano ya que el agua constituye un recurso imprescindible para el desarrollo de las actividades humanas. A su vez, los ecosistemas acuáticos, constituyen en definitiva los colectores de las actividades agrícolas e industriales y desagües de las ciudades.

Entre las comunidades acuáticas se destacan los invertebrados bentónicos que son utilizados habitualmente como bioindicadores para determinar el estado de salubridad del sistema. Dentro de los macroinvertebrados, el grupo taxonómico de mayor diversificación y que mejor los representa es el de los insectos (VIDAL ABARCA GUTIÉRREZ et al., 1994). Si bien los insectos acuáticos son utilizados como herramientas en la definición del estado de calidad de las aguas de cada sistema, para poder emplearlos es indispensable efectuar estudios previos que nos permitan obtener listas taxonómicas que caractericen cada ambiente, información sobre la distribución de los diversos taxa y requerimientos ambientales de los mismos. Sólo de esta manera se puede planear la conservación de los recursos naturales y construir índices bióticos que permitan evaluar el estado de calidad de cada sistema.

El estado actual de conocimiento de la diversidad de la fauna de insectos acuáticos en Argentina es considerable, existiendo numerosas investigaciones realizadas en diferentes regiones del país 
(CORIGLIANO et al., 1996; ALBARIÑO, 1997; DOMÍNGUEZ \& FERNÁNDEZ, 1998; MANGEAUD,1999; MISERENDINO, 2001; MEDINA \& PAGGI, 2004; PAGGI, 2003; PRINCIPE \& CORIGLIANO, 2006; RODRIGUES CAPÍTULO et al., 2002). Para la provincia de Mendoza en Argentina, existen muy pocos registros de estudios limnológicos efectuados a excepción de los realizados por PEŃAFOR (1981), PERALTA (2001) y FUENTES et al. (2004); cabe destacar que ninguno de ellos fue realizado utilizando la fauna de insectos acuáticos.

El estado de desconocimiento de la entomofauna acuática existente en el área de estudio lleva a realizar una prospección minuciosa de los diferentes ambientes (lóticos y lénticos) para poder evaluar la composición faunística de la comunidad invertebrada de las zonas áridas y de esta manera observar si existe un cambio en la riqueza taxonómica a nivel altitudinal y ambiental. Este trabajo es de fundamental importancia ya que exhibe un panorama general de la distribución de las comunidades de insectos acuáticos halladas en Mendoza (Argentina).

\section{MATERIAL Y MÉTODOS}

La provincia de Mendoza, situada al pie de la cordillera de los Andes, se extiende en el centrooeste argentino entre los $32^{\circ}$ y $37^{\circ} 35^{\prime}$ de latitud sur y los $66^{\circ} 30^{\prime}$ y $70^{\circ} 35^{\prime}$ de longitud oeste con una superficie de $150.839 \mathrm{~km}^{2}$. Presenta un clima típicamente templado caracterizado por una extrema aridez, máxima continentalidad y con precipitaciones irregulares y esporádicas generalmente con características de tipo nival.

Se estudiaron dos tipos de ambientes: lótico y léntico. El tipo de ambiente lótico estuvo ubicado en la cuenca del río Mendoza localizada en el noroeste de la provincia de Mendoza (320 49' de latitud sur; $69^{\circ} 57^{\prime}$ y $68^{\circ} 21^{\prime}$ de longitud oeste), mientras que el tipo de ambiente léntico en el Bańado de Carilauquen y Laguna de Llancanelo (350 $00^{\prime}$ y 36 30' de latitud sur; $70^{\circ} 00^{\prime}$ y $68^{\circ} 30^{\prime}$ de longitud oeste) situado en el suroeste de Mendoza. La vegetación típica que rodea el río Mendoza está compuesta por: vegetación altoandina, estepas arbustivas y monte, mientras que la del bañado Carilauquen y laguna de Llancanelo por estepas halófilas.

En el río Mendoza se seleccionaron 11 sitios representativos de diferentes altitudes, composición del sustrato y variables hidrológicas, diez de los cuales pertenecen al ecosistema lótico (Nacientes: cuatro sitios de muestreo; Tramo medio: dos sitios; Tramo inferior: cuatro sitios) y el último sitio (Desembocadura) al ecosistema léntico. En el Bañado Carilauquen se establecieron diez sitios de muestreo siguiendo un gradiente longitudinal desde el inicio del bañado hasta la Laguna de Llancanelo: Nacientes (dos sitios); Tramo medio (cuatro sitios); Tramo inferior (dos sitios); Desembocadura (un sitio) y Laguna (un sitio) (Figura 1.1).

Los muestreos fueron realizados estacionalmente durante un ciclo anual (2000-2001) en el Bañado Carilaunquen y Laguna de Llancanelo, y dos ciclos anuales en la cuenca del río Mendoza (2000-2002). Para ello, se empleó red Surber para los sitios ubicados en el río Mendoza (excepción la desembocadura) y draga de Petersen para la zona de sedimentos blandos correspondiente al Bañado Carilauquen, Laguna de Llancanelo y la desembocadura del río Mendoza. Las muestras fueron conservadas en el campo con alcohol $90 \%$ hasta su posterior procesamiento en el laboratorio. En cada sitio de muestreo se procedió a llenar una planilla con datos tipológicos del ambiente, las siguientes variables fueron determinadas in situ: $\mathrm{pH}$, conductividad, tipificación del sustrato, velocidad de corriente (ambiente lótico), temperatura del agua, temperatura del aire, profundidad del margen muestreado (ambiente lótico), caudal (ambiente lótico) y vegetación dominante.

Para el cálculo de la diversidad, se aplicó el índice de diversidad de Shannon basado en la abundancia proporcional de las especies (MAGURRAN, 1988), por ser el más utilizado en ecología de comunidades. Presenta valores que generalmente oscilan entre 1,5 y 3,5 y tiene la ventaja de distribuirse normalmente, lo que permite realizar comparaciones entre diferentes hábitats, especialmente cuando se trabaja con réplicas. El índice de diversidad de Shannon se calculó con la ecuación: 


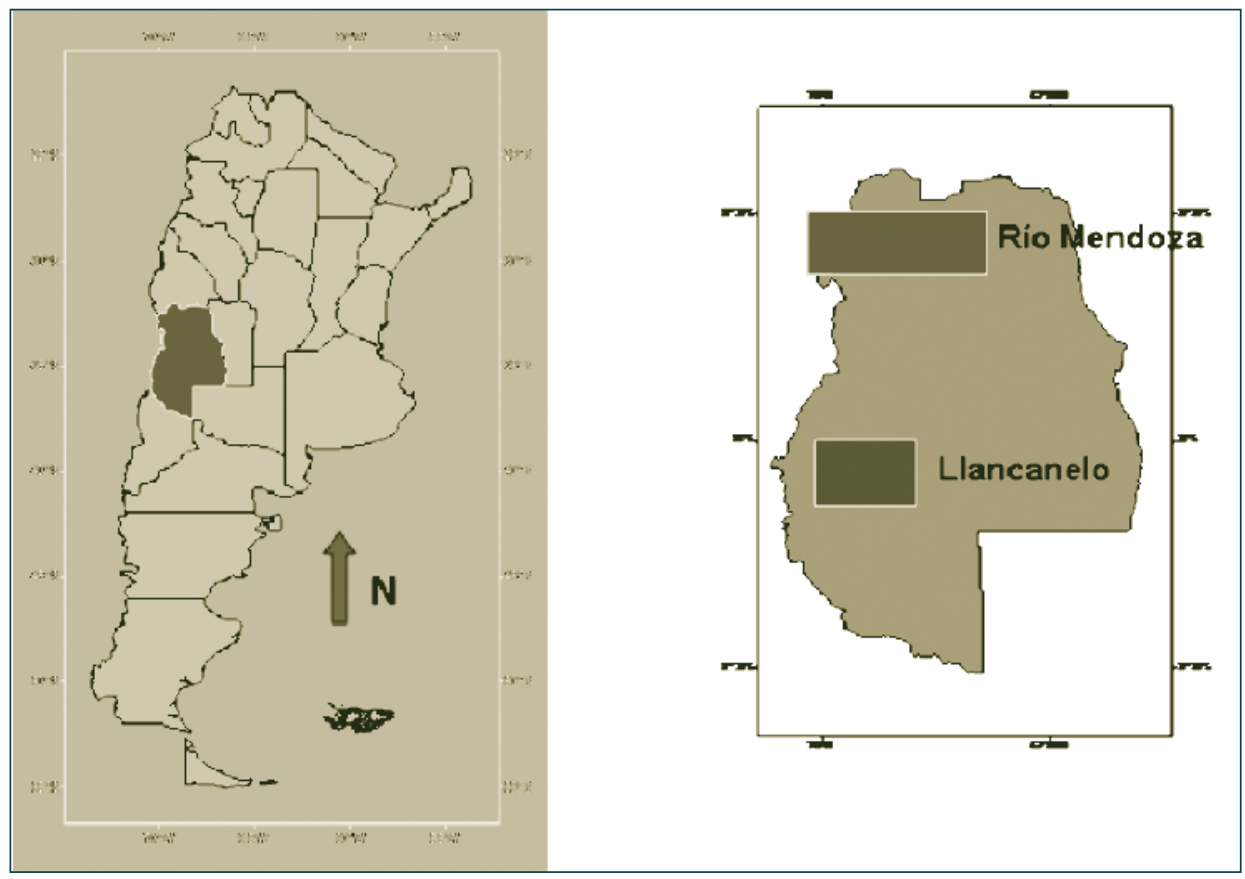

Figura 1. Ubicación del río Mendoza, Bañado de Carilauquen y Laguna de Llancanelo en la provincia de Mendoza, Argentina.

$$
H^{\prime}=-\sum p_{i} \ln p_{i}(p i=n i / N)
$$

$\mathrm{p}_{\mathrm{i}}=$ proporción de la especie “i” en la comunidad.

donde:

pi: proporción del número total de individuos de la especie i en la comunidad.

ni: $n^{\circ}$ de individuos de la especie i de la muestra. $\mathrm{N}: \mathrm{n}^{\circ}$ total de individuos de la muestra.

\section{RESULTADOS}

\section{Condiciones ambientales}

Las principales características ambientales de los sistemas estudiados se presentan en la Tabla 1.

En el Bañado Carilauquen la conductividad aumenta progresivamente hacia su desembocadura alcanzando valores elevados en la desembocadura y la Laguna de Llancanelo (entre 8000 y $19500 \mu \mathrm{Scm}^{-1}$ ) y los mínimos en las nacientes del bañado $\left(930 \mu \mathrm{Scm}^{-1}\right)$. $\mathrm{El} \mathrm{pH}$ varia entre neutro y alcalino dependiendo de los sitios de muestreo. El rango térmico anual registrado varía entre $6.5^{\circ} \mathrm{C}$ de mínima y $25^{\circ} \mathrm{C}$ de máxima dependiendo de la estación climática y del sitio de muestreo; mientras que el sustrato está compuesto por arena y arcilla.

En el río Mendoza los valores de conductividad son elevados, presentando una mineralización excesiva, lo cual se debe principalmente a la tipología del sustrato que atraviesan sus aguas (ARMANDO, 1985). En general los valores de conductividad tienen un rango que oscila entre los 500 y 1500 $\mu \mathrm{Scm}^{-1}$, exhibiendo los sitios de cabecera y desembocadura del río las mayores conductividades. $\mathrm{Al}$ igual que en Llancanelo los valores de $\mathrm{pH}$ varían entre valores neutros y alcalinos de acuerdo a la categorización realizada por NISBET \& VERNEAUX (1970). El rango térmico bianual promedio del agua registrada en los sitios de muestreo del río Mendoza varia entre $2^{\circ} \mathrm{C}$ de mínima y $25,4^{\circ} \mathrm{C}$ de máxima. La transparencia disminuye marcadamente de otoño a verano; la época de aguas claras tuvo lugar en las estaciones climáticas de otońo e invierno. El sustrato que predomina usualmente 
Tabla 1. Características ambientales de los sistemas estudiados.

Valores medios anuales (con máximas y mínimas).

\begin{tabular}{|c|c|c|}
\hline Características generales & Río Mendoza & Bańado Carilauquen y Laguna \\
\hline Latitud & S 32० 49' & S $35^{\circ}$ y $36^{\circ} 30^{\prime}$ \\
\hline Longitud & W $69^{\circ}$ y $68^{\circ} 21^{\prime}$ & W $70^{\circ}$ y $68^{\circ} 30^{\prime}$ \\
\hline Altitud promedio (m.s.n.m) & $2800-600$ & 1280 \\
\hline Sustrato dominante & bloque-canto rodado-guijarro & arena-arcilla \\
\hline Vegetación dominante & Corteria rudiuscula & $\begin{array}{l}\text { Scirpus californicus } \\
\text { Corteria rudiuscula }\end{array}$ \\
\hline Uso de la tierra & rural-urbano & rural \\
\hline $\mathrm{pH}$ & $\begin{array}{r}8,25 \\
10,2-6,61\end{array}$ & $\begin{array}{r}7,42 \\
6,48-8,90\end{array}$ \\
\hline Conductividad $\left(\mu \mathrm{S} . \mathrm{cm}^{-1}\right)$ & $\begin{array}{r}1047,83 \\
5290-364 \\
\end{array}$ & $\begin{array}{r}3243 \\
914-19520 \\
\end{array}$ \\
\hline Temperatura del agua $\left({ }^{\circ} \mathrm{C}\right)$ & $\begin{array}{r}9,30 \\
25,40-1,90 \\
\end{array}$ & $\begin{array}{r}15,93 \\
6,50-25,60 \\
\end{array}$ \\
\hline Temperatura del aire $\left({ }^{\circ} \mathrm{C}\right)$ & $\begin{array}{r}15,97 \\
36-2 \\
\end{array}$ & $\begin{array}{r}15,21 \\
4-30,80 \\
\end{array}$ \\
\hline Transparencia (m) & $\begin{array}{r}0,12 \\
0,4-0,01 \\
\end{array}$ & $\begin{array}{r}0,60 \\
0,20-2 \\
\end{array}$ \\
\hline
\end{tabular}

en la mayoría de los sitios de muestreo es bloque pequeńo, canto rodado, guijarro y arena-limo.

\section{Composición faunística}

\section{Bañado Carilauquen y Laguna de Llancanelo}

La comunidad de insectos acuáticos está compuesta por seis órdenes: Diptera, Trichoptera, Ephemeroptera, Odonata, Hemiptera y Coleoptera; siendo Diptera (15 taxones) y Coleoptera (14 taxones) los órdenes en los que se observan mayor riqueza de taxones (Anexo 1). El bañado mantiene un número constante de taxones en las nacientes y sector medio del sistema, para luego disminuir abruptamente en la desembocadura del bañado y Laguna de Llancanelo.

Dentro del orden Diptera, la familia Chironomidae es el grupo taxonómico que tiene mayor frecuencia de aparición a nivel espacial y estacional y mayor riqueza taxonómica comparando con el resto de la comunidad de insectos acuáticos estudiada. Está representada por tres subfamilias: Orthocladiinae, Tanypodinae y Chironominae, siendo Chironomi- nae la subfamilia que presenta mayor riqueza genérica y densidad. Si bien los géneros Dasyhelea Kieffer (Ceratopogonidae) y Metrichia (Hydroptilidae) le siguieron en abundancia a los quironómidos, éstos no tienen la misma representatividad en el gradiente longitudinal del sistema.

Del análisis del ciclo anual se observa que ciertos taxones fueron representativos de cada sitio de muestreo. Las nacientes del bañado están representadas por Cricotopus, Metrichia y Oxyethira; siendo estos géneros exclusivos del sector. El tramo medio dispone de un mayor número de grupos taxonómicos contemplando una mayor riqueza genérica de coleópteros, como así también se registran odonatos (Aeshna), hemípteros (Belostoma, Notonecta viscerens y Corixidae), quironómidos (Tanypus, Chironomus y Pseudochironomus) y ceratopogónidos (Dasyhelea). En el tramo inferior se hallan los géneros Chironomus, Paratanytarsus, Djalmabatista y Tanypus. Mientras que en la desembocadura y la laguna disponen de un menor número de grupos taxonómicos: Chironomus, Tabanidae, Staphylinidae y Berosus. 


\section{Anexo 1}

Lista taxonómica de los insectos acuáticos hallados en el bañado Carilauquen y laguna de Llancanelo durante el ciclo anual (período 2000/2001).

\section{INSECTA}

\section{DIPTERA}

Chironomidae

Subfamilia Orthocladiinae

Cricotopus

Subfamilia Tanypodinae

Tanypus

Djalmabatista

Subfamilia Chironominae

Polypedilum

Chironomus

Tanytarsus

Pseudochironomus

Paratanytarsus

Dicrotendipes

Ceratopogonidae

Dasyhelea

Culicidae

Culex

Ephydridae

Tabanidae

Dolichopodidae

Dixidae

\section{COLEOPTERA}

Hydrophilidae

Berosus sp1

Berosus sp2

Tropisternus setiger

Enochrus lampros

Dysticidae
Liodessus
Lancetes biremis
Lancetes varius
Laccornellus
Hydrovatus
Desmopachria mendozana
Haliplidae
Haliplus
Curculionidae
Stenopelmus minutus
Carabidae
Subtribu Bembidiina
Staphylinidae
ODONATA
Aeshnidae
Aeshna
TRICHOPTERA
Hydroptilidae
Metrichia
Oxyethira
HEMIPTERA
Notonectidae
Notonecta viscerens
Belostomatidae
Belostoma
Corixidae

Dysticidae

Lancetes biremis

Hydrovatus

Desmopachria mendozana

Haliplidae

Haliplus

Stenopelmus minutus

Carabidae

Subtribu Bembidiina

ODONATA

Aeshnidae

TRICHOPTERA

Metrichia

\section{Río Mendoza}

En el río Mendoza se hallan cinco órdenes de insectos acuáticos: Plecoptera, Ephemeroptera, Trichoptera, Coleoptera y Diptera. El orden Diptera (25 taxones) presenta mayor riqueza taxonómica, densidad y frecuencia de aparición a lo largo del perfil longitudinal del río; siguiéndole en orden decreciente de densidad Trichoptera (tres taxones), Ephemeroptera (tres taxones), Coleoptera (tres taxones) y Plecoptera (un taxón) (Anexo 2).
La familia Chironomidae presenta la mayor abundancia relativa y riqueza genérica en los 11 sitios de muestreo. Está representada por cuatro subfamilias: Orthocladiinae, Podonominae, Tanypodinae y Chironominae. A diferencia de lo hallado en el bañado Carilauquen y laguna de Llancanelo la subfamilia Orthocladiinae es la predominante y la que tiene mayor riqueza genérica en casi todos los sitios de muestreo siendo reemplazada por Chironominae en la desembocadura del sistema. 


\section{Anexo 2}

Lista taxonómica de los insectos acuáticos hallados en el río Mendoza durante el ciclo bianual (período 2000-2002).

\section{PLECOPTERA}

Gripopterygidae

Limnoperla sp.

\section{EPHEMEROPTERA}

Baetidae

Baetodes sp.

Andesiops sp.

Leptophlebiidae

Massartellopsis sp.

TRICHOPTERA

Hydrobiosidae

Cailloma lucidula

Hydropsychidae

Smicridea murina

Familia Hydroptilidae

Metrichia neotropicalis

\section{COLEOPTERA}

Elmidae

Austrelmis sp.

Hydrophilidae

Berosus sp.

Staphylinidae

Thinobius sp.

\section{DIPTERA}

Athericidae

Atherix variegata

Blephariceridae

Edwarsina sp.

Ceratopogonidae
A lo largo del perfil longitudinal del río, se observa un reemplazo en los taxa encontrados entre los sitios de cabecera, las halladas en el tramo intermedio y en la desembocadura, y se puede observar marcadamente esta sustitución a través de ciertos géneros en los órdenes Ephemeroptera, Trichoptera, Coleoptera y Diptera.

En el caso de las efémeras (Ephemeroptera), se puede observar como el género Andesiops abundante en las cabeceras, es reemplazado por Baetodes en el tramo medio e inferior. Si bien Massartellopsis presenta una densidad baja comparada con los otros dos géneros, sirve como demostración del reemplazo existente en el gradiente longitudinal, ya que al igual que Andesiops se halla únicamente en las cabeceras.

Con los tricópteros, se muestra un patrón marcado en Smicridea murina. Esta especie, ausente en las cabeceras, es encontrada en el tramo medio aumentando paulatinamente su densidad hacia el tramo inferior. Cailloma lucidula es característica de las cabeceras y tramo medio disminuyendo su densidad hasta hacerse nula en el tramo inferior.

El tramo medio muestra la mayor riqueza taxonómica encontrándose tanto taxones pertenecientes a la cabecera del sistema como al tramo inferior considerándose una zona de transición entre los tramos altos y bajos del río.

En ambos sistemas estudiados se observa una riqueza taxonómica y diversidad (Figura $2 \mathrm{a}$ y b) muy baja si se la compara con otros sistemas lóticos y lénticos de Argentina (CORIGLIANO et al., 1996; RODRIGUES CAPÍTULO, 2001; MEDINA et al., 2004; FONTANARROSA et al., 2004; MISERENDINO, 2001; PRÍNCIPE et al., 2006. Llancanelo presenta una diversidad menor que el río Mendoza, con un valor promedio máximo de 0,5 en el tramo medio y un valor promedio mínimo de 0,21 en el tramo inferior; mientras que el río Mendoza exhibe un máximo de 1,57 y un mínimo de 0,015 . 


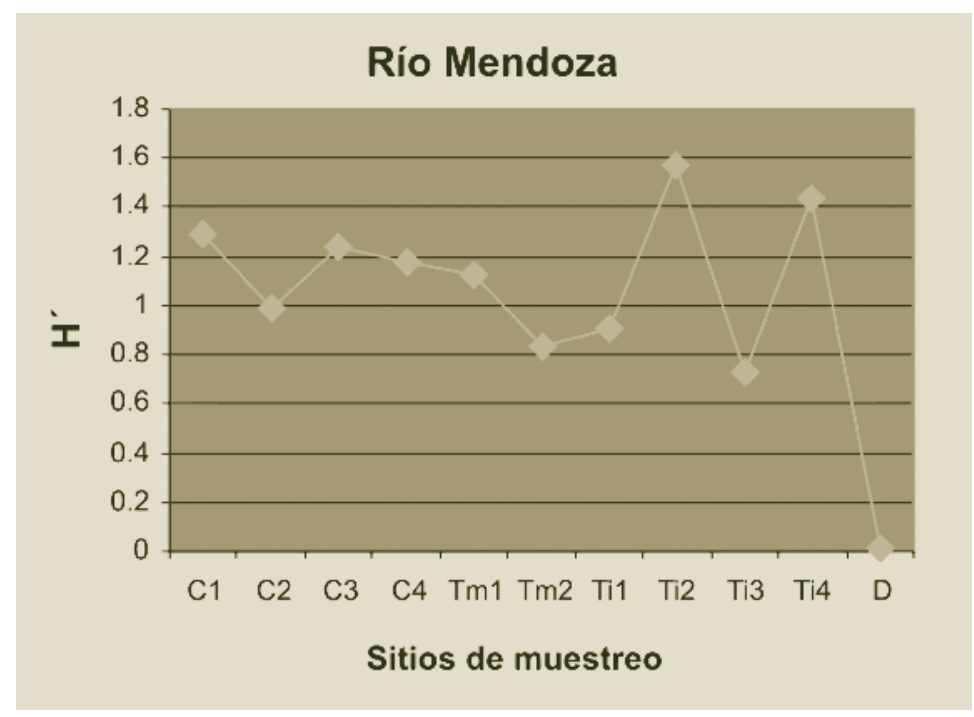

A

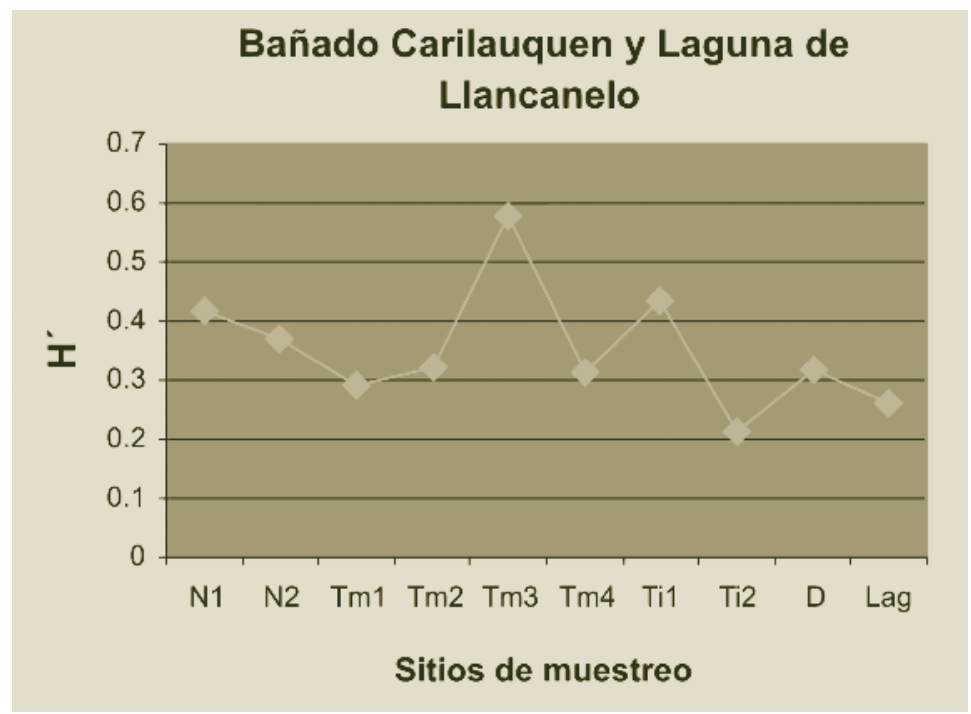

B

Figura 2 A, B. Representación gráfica del indice de diversidad de Shannon en el río Mendoza y Bañado de Carilauquen.

\section{CONCLUSIONES}

A pesar que los ambientes estudiados poseen diferencias latitudinales, altitudinales, geomorfológicas, y ambientales, en la composición de las comunidades acuáticas halladas hemos encontrado las siguientes similitudes:

- Los sistemas acuáticos presentaron valores de diversidad bajos.
- La mayor riqueza taxonómica se halló en los tramos medios de ambos sistemas.

- El orden Diptera es predominante tanto en riqueza, densidad como en representatividad a lo largo del gradiente ambiental tanto en el río Mendoza como en el bañado y laguna de Llancanelo.

- La familia Chironomidae tuvo mayor frecuencia de aparición, riqueza genérica y densidades en los ambientes lóticos y lénticos. 
- La abundancia y distribución de los insectos acuáticos en los sistemas estudiados, están afectadas principalmente por la heterogeneidad espacial.

- Los valores de conductividad fueron elevados y el pH osciló entre neutro y alcalino.

\section{BIBLIOGRAFÍA}

ARMANDO V. 1985. Hidroquímica. Consejo Profesional de Ingenieros y Geólogos. 13-16.

ALBARIÑO, R. J. 1997. Spatial distribution of Plecoptera from an Andean-Patagonic lotic environment in Argentina. Rev. Brasil. Biol. 57: 629-636.

CORIGLIANO, M. C, C. M. GUALDONI, A. M. OBERTO, \& G. B. RAFFAINI. 1996. Macroinvertebrados acuáticos de Córdoba. Biodiversidad de la Provincia de Córdoba. Fauna. Vol. 1: 119-165.

DOMÍNGUEZ, E. \& H. R. FERNÁNDEZ. 1998. Calidad de los Ríos de la Cuenca Salí (Tucumán, Argentina) medida por un índice biótico. Serie Conservación de la Naturaleza 12: 1-40.

FONTANARROSA M. S., P. L. M. TORRES, \& M. C. MICHAT. 2004. Comunidades de insectos acuáticos de charcos temporários y lagunas en la ciudad de Buenos Aires (Argentina). Rev. Soc. Entomol. Argent. 63 (3-4): 55-65.

FUENTES, V. 2000. Variación diaria del plancton en un río de Alta Cordillera (Uspallata, Mendoza). Trabajo de la Tesis de Grado de la Fac. Ciencias Exactas y Naturales, Córdoba. 80 p.

MANGEAUD, A. 1999. Efectos de Fuentes de contaminación puntual sobre las comunidades de insectos bentónicos en la cuenca del Suquía (Córdoba, Argentina). Rev. Soc. Entomol. Argent. 58 (1-2): 218-222.

MEDINA, A. I. \& A. C. PAGGI. 2004. Composición y abundancia de Chironomidae (Diptera) en un río serrano de zona semiárida (San Luis, Argentina). Rev. Soc. Entomol. Argent. 63 (3-4): 107-118.

MISERENDINO, M. L. 2001. Macroinvertebrate assemblages in Andean Patagonian rivers and stream: environmental relationships. Hydrobiologia 444: 147-158.

NISBET, M. \& J. VERNEAUX. 1970. Composantes Quimiques des eaux courantes. Ann. Limnol. 6 (2): 161-190.

PAGGI, A. C. 2003. Los Quironómidos (Diptera) y su empleo como bioindicadores. Biología Acuática 21: 50-57.

PENAFOR MB. 1981. Relevamiento de la ictiofauna y determinación de áreas naturales en dos ríos mendocinos. Boletín del Museo de Ciencias Naturales y Antropológicas No 2.

PERALTA, P. \& C. CLAPS. 2001. Seasonal Variation of the Mountain Phytoplankton in the Arid Mendoza Basin, Westcentral Argentina. J. Freshwater Ecol. 16 (3), 445-454.

PRINCIPE, R. E. \& M. C. CORIGLIANO. 2006. Benthic, drifting and marginal macroinvertebrate assemblages in a lowland river: temporal and spatial variations and size structure. Hydrobiologia 553: 303-317.

RODRIGUES CAPÍTULO, A. 2001. Use of benthic macroinvertebrates to assess the biological status of Pampean streams in Argentina. Aquatic Ecology 35: 109-119.

RODRIGUES CAPÍTULO, A., A. C. PAGGI, \& C. S. OCÓN. 2002. Zoobenthic communities in relation to slope, substrate heterogeneity and urban disturbances in Pampean hill streams (Argentina). Verh. Internat. Verein. Limnol. 28: 1267-1273.

VIDAL ABARCA GUTIERREZ, M. R., M. L. SUÁREZ ALONSO, R. GÓMEZ CEREZO, \& L. RAMÍREZ-DÍAZ. 1994. Ecología de Aguas Continentales. Prácticas de Limnología. Universidad de Murcia. 266 p. 